
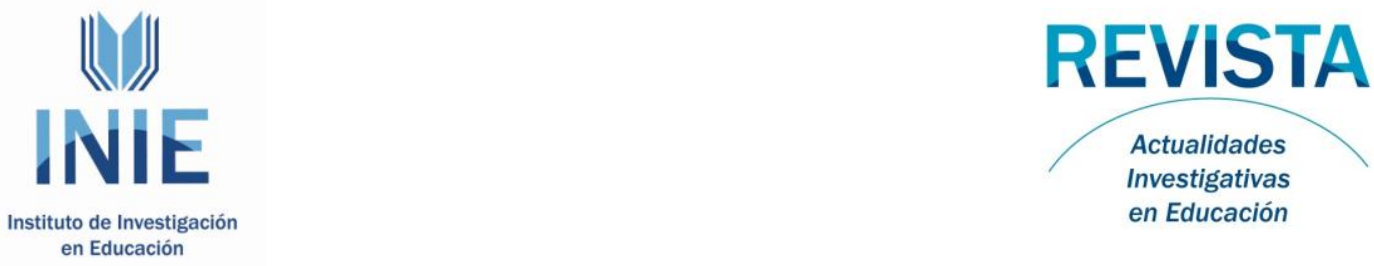

Actualidades Investigativas en Educación

Revista Electrónica publicada por el

Instituto de Investigación en Educación

Universidad de Costa Rica

ISSN 1409-4703

http://revista.inie.ucr.ac.cr

COSTA RICA

\title{
LAS BITÁCORAS ELECTRÓNICAS, O BLOGS, Y SU UTILIZACIÓN PARA EL DESARROLLO DE LA COMPETENCIA COMUNICATIVA: SÍNTESIS DE UNA EXPERIENCIA EN LA FORMACIÓN DE DOCENTES DE EDUCACIÓN PRIMARIA
}

ELECTRONIC BLOGS AND THEIR EFFECTIVENESS FOR THE DEVELOPMENT OF COMMUNICATION COMPETENCE: SYNTHESIS OF AN EXPERIENCE IN TRAINING PRIMARY SCHOOL TEACHERS

Volumen 10, Número 2

pp. 1-14

Este número se publicó el 30 de agosto de 2010

Olga Ligia Solano Córdoba

La revista está indexada en los directorios:

LATINDEX, REDALYC, IRESIE, CLASE, DIALNET, DOAJ, E-REVIST@S,

La revista está incluida en los sitios:

REDIE, RINACE, OEI, MAESTROTECA, PREAL, HUASCARAN, CLASCO 


\title{
LAS BITÁCORAS ELECTRÓNICAS, O BLOGS, Y SU UTILIZACIÓN PARA EL DESARROLLO DE LA COMPETENCIA COMUNICATIVA: SÍNTESIS DE UNA EXPERIENCIA EN LA FORMACIÓN DE DOCENTES DE EDUCACIÓN PRIMARIA
}

\author{
ELECTRONIC BLOGS AND THEIR EFFECTIVENESS FOR THE DEVELOPMENT OF \\ COMMUNICATION COMPETENCE: SYNTHESIS OF AN EXPERIENCE IN TRAINING \\ PRIMARY SCHOOL TEACHERS
}

\section{Olga Ligia Solano Córdoba ${ }^{1}$}

\begin{abstract}
Resumen: En este artículo, a partir del análisis del impacto del Internet en los procesos de aprendizaje y en la vida cotidiana de la juventud costarricense, se expone una experiencia en la utilización de bitácoras electrónicas o "blogs" para el desarrollo de la competencia comunicativa. Esta experiencia consistió en la creación de bitácoras electrónicas o "blogs", en forma grupal, para la elaboración y difusión de una indagación sobre varias temáticas de la realidad centroamericana. Se llevó a cabo durante el primer semestre de 2008 con estudiantes del curso Expresión Escrita I perteneciente al Bachillerato en Educación Primara de la Escuela de Formación Docente de la Universidad de Costa Rica. A partir de la experiencia descrita, se promueve la reflexión y la inquietud sobre las posibilidades que brinda Internet, específicamente la Web 2.0, para el desarrollo de la competencia comunicativa. Se concluye que la utilización de bitácoras electrónicas o "blogs", permitió innovar procesos educativos y generar contextos significativos para el desarrollo de la competencia comunicativa.
\end{abstract}

Palabras clave: LA INTERNET Y EL APRENDIZAJE, WEB 2.0, BITACORAS ELECTRÓNICAS, ENFOQUE COMUNICATIVO, COMPETENCIA COMUNICATIVA

Abstract:

Stemming from an analysis of the impact of Internet on the learning process and on the daily life of Costa Rican youth, this paper portrays an experience in the use of electronic blogs for the development of communication competence. The study was conducted generating group blogs to explore and disseminate different topics of the Central American reality; it was conducted during the first semester of the 2008 academic year, with students of the Written Expression I course of the Bachelor's in Elementary Education of the School of Teacher Education at the University of Costa Rica. The knowledge developed from this practice created awareness and encouraged reflection on the possibilities offered by Internet and specifically the Web 2.0. The paper concludes that the use of electronic blogs in this instance, promoted innovation of the educational process and generation of meaningful contexts for the development of communication competence.

Key words: INTERNET AND LEARNING, WEB 2.0, ELECTRONIC BLOGS, COMMUNICATION APPROACH, COMMUNICATION COMPETENCE

\footnotetext{
Doctora en Estudios Latinoamericanos y Máster en Informática Educativa ambos títulos de la Universidad Nacional de Costa Rica. Profesora Adjunta de Escuela Ciencias del Lenguaje del Instituto Tecnológico de Costa Rica.
}

Dirección electrónica: olsolano@itcr.ac.cr

Artículo recibido: 7 de mayo, 2010

Aprobado: 12 de julio, 2010 


\section{Introducción}

La internet y sobre todo la World Wide Web han significado un importante aporte para la enseñanza y el aprendizaje de la sociedad en general y en particular de la juventud. Castells (2006) afirma es que a partir de 1996, aproximadamente, cuando la "www" fue aplicada por primera vez a la enseñanza, y los profesores comenzaron a incorporar la internet a sus prácticas educativas. Entonces, se utilizó para ilustrar clases presenciales a través de páginas web, realizar debates en línea para continuar con la discusión de una clase o conferencia, asignar temas para investigar en la web o proporcionar cuestionarios y otras actividades para complementar los libros de texto utilizados en la clase.

Con Web 1.0, la internet ofrecía al campo educativo los siguientes servicios: correo electrónico, transferencia de archivos, grupos de noticias, listas de interés, conversaciones simultáneas y aplicaciones hipermediales (www); y con la aparición de la Web 2.0, las posibilidades educativas que ofrece la internet no sólo han aumentado, sino que se puede hablar de un cambio de paradigma sobre la concepción de la internet y sus funcionalidades.

En consecuencia, a la Web 1.0 se le ha señalado su marcada unidireccionalidad, mientras que la Web 2.0 se orienta más a facilitar una mayor interacción entre los usuarios, quienes pueden expresarse, opinar, buscar y recibir información de interés, compartir contenidos, así como colaborar en la creación de nuevo conocimiento, en fin, generan colaboración en línea.

Para Serrano (2006), la Web 2.0 ofrece:

- Arquitecturas de información, participativas y democráticas.

- Paso de una comunicación unidireccional (un autor emite, los usuarios reciben) a una comunicación multidireccional (todos emiten, todos reciben).

- Empleo de software libre y bajas inversiones, producto de un mercado que ha sobrevivido a la burbuja de la internet.

- Uso de aplicaciones a través de la web, como editores de texto y otros.

Para Hernández (2007) la internet nunca había sido pensada por y para la gente. Había sido pensada como un campo de trabajo y acción para aquellos que poseían conocimientos en informática. Sin embargo, con la aparición de la Web 2.0 empieza a ser más consecuente hablar de democracia en la internet, de la distribución de información y el conocimiento por canales informales, así como de la posibilidad de modificar y de adaptar los productos que existen en el mercado a las necesidades particulares. 
Por lo anterior, se cuestiona: ¿cuál es el uso que le estamos dando a las TIC, específicamente a la internet y a las posibilidades que brinda la Web 2.0, en nuestras aulas? A continuación, planteo mis reflexiones sobre la experiencia de Costa Rica al respecto, y expongo una experiencia desarrollada con el uso de las bitácoras electrónicas, o "blogs", como estrategia para el desarrollo de la competencia comunicativa.

\section{DESARROLLO}

\subsection{Antecedentes sobre la incorporación de las TIC en la educación pública costarricense}

Desde finales de los años 80, específicamente en el año 1988, inicia en Costa Rica la incorporación de las computadoras en la educación pública costarricense, se empiezan a utilizar desde el primer hasta el noveno año de la educación general básica. Posteriormente, surge la posibilidad de conectarlas a la internet.

De este modo, y al igual que en el resto del mundo, se espera que con las nuevas tecnologías de la información y la comunicación incorporadas a las prácticas escolares se logre elevar la calidad de nuestra educación, así como evitar la deserción escolar. Al respecto escribe Fonseca (2000) “...nuestro Programa ${ }^{2}$ fue puesto en marcha justamente con el objetivo de reanimar la vida educativa del país y de ofrecer oportunidades de crecimiento personal y académico a niños y maestros".

Sin embargo, pareciera que reanimar la vida educativa del país en escuelas y colegios, no ha sido posible. En el XIV Informe sobre el Estado de la Nación (2008) se afirma lo siguiente

Costa Rica tiene dificultades para lograr que los niños y niñas culminen con éxito su paso por el sistema educativo. Los datos de seguimiento de cohortes de 1997 al 2007 indican que, en primaria, entre el $85 \%$ y el $90 \%$ de los alumnos que ingresó a primer grado logró terminar el nivel, aunque de ellos solo entre el 50\% y el 58\% lo hizo sin repetir ningún año. En secundaria la eficiencia es aún menor: apenas una cuarta parte

2 Se refiere al Programa Nacional de Informática Educativa MEP-FOD, que es una iniciativa nacional conjunta del Ministerio de Educación Pública de Costa Rica y de la Fundación Omar Dengo. El programa que se lleva que a cabo desde 1988 y beneficia a un significativo grupo de población estudiantil, que va desde el preescolar hasta el noveno año de la Educación General Básica, en un rango de edades de cinco a quince años, aproximadamente. 
de los estudiantes que inician en sétimo año consigue graduarse. EI MEP estima que de cada mil niños de la cohorte del 2007, solo 381 se graduarán.

Esta información refleja que continúan creciendo los niveles de deserción escolar, específicamente, en escuelas y colegios. Además, el rendimiento está muy por debajo de lo esperado; al respecto, los y las jóvenes manifiestan que una de las razones por las cuales desertan de las aulas es que el colegio es muy aburrido y que no les enseñan las cosas que necesitan saber para poder enfrentar la vida.

En ese sentido, el personal docente manifiesta frustración, porque la juventud no pareciera tener interés por aprender, se estudia solo para los exámenes y una vez que estos pasan ya no recuerdan los contenidos; al mismo tiempo, la cantidad de personas que optan por estudiar en forma independiente crece, por ejemplo, en los últimos años se ha registrado un considerable aumento en la cantidad de personas que asiste a programas de Educación Abierta, así como la cantidad de graduados por esta modalidad, que no implica, necesariamente, asistir a un centro educativo, ya que el o la estudiante puede prepararse en forma individual, con un profesor tutor o asistir a proyectos privados o públicos que imparten la modalidad.

Uno de los objetivos relacionados con la introducción de tecnologías de la información y la comunicación en ambientes educativos es potenciar los procesos de aprendizaje; sin embargo, si analizamos el uso de estas en las aulas escolares podríamos decir que, en su mayoría, se limitan sobre todo al uso de presentaciones multimedia para apoyar exposiciones y conferencias.

Precisamente, un avance de resultados presentado por el Programa Sociedad de la Información y el Conocimiento de la Universidad de Costa Rica (PROSIC, 2008a), acerca de una investigación sobre: "Los jóvenes costarricenses en la Sociedad de la Información", realizada con estudiantes universitarios y de segunda enseñanza entre mayo y diciembre de $2007^{3}$, nos muestra una de sus conclusiones

El uso de TIC durante el tiempo de clases está aumentando ya que por ejemplo el uso de presentaciones en Power Point alcanza cerca del 40\%. No así para el uso de la internet que en promedio es utilizado en un $4,2 \%$ de las lecciones. (p. 63)

3 A la fecha, el Programa Sociedad de la Información y el Conocimiento de la Universidad de Costa Rica (PROSIC) presentó el informe final de la investigación; sin embargo, se toma esta cita del avance parcial, ya que en el informe final se refiere al respecto en forma más general. 
En el caso de la educación superior costarricense, es en esta década, la primera mitad del siglo XXI, cuando se inicia con la implementación de entornos virtuales de aprendizaje (EVA) como una posibilidad para incorporar la internet en los procesos de enseñanza y aprendizaje; estas permiten la creación de cursos virtuales, desde la exposición de contenidos, actividades para el aprendizaje de esos contenidos: foros, debates en línea, así como actividades de evaluación: exámenes, elaboración de ensayos, etcétera.

En el caso del Instituto Tecnológico de Costa Rica (ITCR) se cuenta, desde hace aproximadamente cinco años, con una plataforma virtual que tiene como objetivo la integración de las tecnologías de información y comunicación a los procesos de enseñanzaaprendizaje; asimismo, se ha incentivado la implementación de cursos bimodales y cursos virtuales como parte de la oferta educativa de esta institución. En la misma línea han trabajado la Universidad Nacional a través de su programa UNA VIRTUAL y la Universidad de Costa Rica a través de METICS, una Unidad de la Vicerrectoría de Docencia, así como la Universidad Estatal a Distancia (UNED) con un Programa de Aprendiza en Línea.

Con la puesta en marcha de estas iniciativas se ha empezado a poner en práctica, en las universidades estatales costarricenses, la: "enseñanza reforzada con la web o con la internet'. En algunos casos se han reducido las clases presenciales para permitir un mayor aprendizaje digital, también conocido como híbrido, combinado o distribuido.

Por lo tanto, tenemos que en nuestro país, tanto en la educación primara, secundaria como en la universitaria, la internet forma parte de los escenarios posibles para el aprendizaje.

\subsection{Uso de las TIC por parte de la juventud costarricense}

El Programa de la Sociedad de la Información (PROSIC), de la Universidad de Costa Rica (UCR), en su Informe del año 2008 dedica su capítulo No.8 al tema e-Juventud en Costa Rica; este fue elaborado a partir de una encuesta realizada a nivel nacional entre mayo y noviembre del 2007, que se aplicó en forma personal a 4,037 jóvenes de colegios y de universidades públicas y privadas con edades comprendidas entre 12 y 24 años. Del mismo se extrae la siguiente información:

- La gran mayoría de jóvenes utiliza, prioritariamente, el correo electrónico (67,4\%), la internet $(61,7 \%)$ y programas de música $(54,9 \%)$. 
- Los y las jóvenes en esta encuesta se perciben en un 41,8\% como la persona que más sabe sobre el uso de la computadora y la internet en su familia.

Entre los y las jóvenes el proceso de aprendizaje, por medio de los amigos, es muy importante y esta práctica adquiere mayor relevancia cuando se trata de las TIC: las formas más frecuentes de adquisición de conocimientos sobre estas han sido mediante ayuda de los amigos, con un 51,2\%; por sí mismos un 43,3\%, y la familia un $32,2 \%$. El lugar donde los y las jóvenes han sido más motivados para utilizar las tecnologías es en su casa, con casi el $40 \%$ de los casos, y en los centros de enseñanza con un $34 \%$.

En detalle, los colegiales han sido motivados más fuertemente en su propia casa, con un $44,4 \%$, que en el colegio, con tan solo un 25,1\%; mientras que en los universitarios la relación es inversa, un $30,8 \%$ en la casa, y $46 \%$ en la universidad. La actividad que más enseñan es a navegar en la internet para un $86 \%$, seguido de quemar discos con un $71,3 \%$, jugar un 60,9\%, y descargar programas un $51,3 \%$.

Se observa que los y las jóvenes que asisten a instituciones educativas privadas tienen mayor conexión a la internet, es decir, un 75,8\% ante un 29,7\%; sin embargo, los que asisten a instituciones públicas buscan alternativas para poder accesar a los equipos y servicios TIC, pues la proporción que usan es superior a la proporción de tenencia de estas en el hogar: en el caso de la internet un $44,2 \%$ afirma tenerla en el hogar, pero su uso equivale a un $89,8 \%$. En ese sentido, la internet aparece como segundo equipo más usado por los jóvenes.

Un $33,5 \%$ de la población encuestada se conecta a la internet más de 10 horas a la semana, mientras que en el grupo de colegiales solamente un $13,4 \%$ lo hace con esa frecuencia.

Los sitios Web más visitados son los buscadores con $82,5 \%$, el correo electrónico $70,6 \%$ y sitios relacionados con la música 52,3\%, seguidos por páginas de perfiles personales como Hi5.com con un 50,1\%; páginas dedicadas a juegos un 34,9\%; Wikipedia.org un 36,1\%, y You Tube un 37,3. Conforme avanzan las edades es más común el uso de los buscadores para un 95,1\%, y el correo electrónico un 91,4\%, y ya no es tan frecuente visitar páginas relacionadas con juegos, pues esta variable baja a un 15,2\%, o las relacionadas con música un $34,2 \%$.

Los usos del correo electrónico son diversos, en su mayoría se afirma que el principal uso que le dan es para enviar y recibir chistes, bromas, noticias, etcétera; no obstante, conforme avanza la edad disminuye esta tendencia; el segundo uso está relacionado con 
actividades académicas y mantenerse en contacto con sus amigos y familiares, fundamentalmente, las mujeres, los grupos de menor edad, y quienes tienen una condición económica alta son quienes más lo usan para este fin; por último, el uso del correo como medio para hacer trabajos del centro educativo es más frecuente entre la población joven de 21 a 24 años de instituciones públicas.

De acuerdo con la variable "sexo" son las mujeres quienes más lo utilizan para estas actividades, así como los de condición económica media. En la población entrevistada se utiliza, con mayor frecuencia, la mensajería instantánea para un 59,1\% y el chat un 50,6\%. En menor grado se realizan llamadas por la internet para un 13,3\%, los foros de discusión un $9,8 \%$, y los blogs $8,1 \%$.

Más recientemente, en abril de 2009, el Instituto de Estudios de Población (IDESPO) de la Universidad Nacional (UNA) realizó una encuesta telefónica, sobre Jóvenes y TICs, a 400 personas entre 15 y 25 años, costarricenses o extranjeras (con dos o más años de residir en el país), residentes en viviendas particulares y con teléfono. El análisis de los resultados de esta encuesta muestra cómo la internet le permite a un 33,3\% de la población joven tomar decisiones importantes en su vida personal; el mismo porcentaje afirma tener confianza en este medio de comunicación y un $52 \%$ se identifica con la expresión: "son apasionantes", cuando piensan en nuevas tecnologías.

De hecho, un $71,3 \%$ asocia el término TIC con medios y aparatos de comunicación. Un $61,5 \%$ opina que la internet ha influido positivamente en sus relaciones personales; un $52,8 \%$ considera que le sirve para despejarse y un $72,0 \%$ considera que la internet le ha cambiado la vida y la forma de relacionarse con los jóvenes.

Con respecto al uso que se le da a la internet, se encontró que un $89 \%$ lo utiliza para búsquedas; un 87,3\% utiliza el correo electrónico; un 84,5\% para consultar documentos; un $70,3 \%$ para escuchar música; un $61,1 \%$ para chatear y participar en foros, y solo un $14,9 \%$ para participar en blogs.

Como vemos, hoy la población joven, en su mayoría, tiene cuenta electrónica, usa los servicios de mensajería instantánea y forma parte de redes sociales como Hi5, Faceebook, MySpace, entre otras; a través de estas, "conversan" con sus amigos y amigas, tienen listas de contactos, amistades virtuales, mantienen contacto con quienes están fuera de nuestras fronteras, y en algunos casos se comunican con esta comunidad en otro idioma, lo que viene a contribuir con la posibilidad de practicar una segunda lengua y conocer otras culturas. 
Un dato curioso es que aunque los estudios nos indican, además, que uno de los usos más frecuentes que se le da a la internet es la búsqueda de información, los profesores universitarios, sobre todo, se quejan de que los trabajos que presentan los estudiantes son plagios o retazos elaborados a partir de "cortar" y "pegar" en documentos bajados de la internet, no se observa lectura analítica, crítica, ni producción personal de los y las estudiantes.

Por su parte, el uso de simulaciones, robótica educativa, videoconferencias y otras aplicaciones de alto potencial educativo es muy limitado en nuestra realidad costarricense. Incluso, las conversaciones sincrónicas y asincrónicas, que podrían ser una fuente para el intercambio de experiencias culturales, es muy utilizada por la juventud, pero no como parte de procesos formales de educación.

¿Por qué será que las TIC no han impactado los procesos de educación formal de la misma manera que lo han hecho en los procesos de educación informal? Quizá la respuesta está en la forma en que estamos usando estas tecnologías en los ambientes educativos formales. No nos hemos dado cuenta que el cambio no solo es de forma, sino de fondo. En ese sentido Margarita Mead (1971) citada por Martín-Barbero $(2002$, ๆ 3) manifiesta que

...nuestro pensamiento nos ata todavía al pasado, al mundo como existía en la época de nuestra infancia y nuestra juventud, nacidos y creados antes de la revolución electrónica, la mayoría de nosotros no entiende lo que esto significa. Los jóvenes de la nueva generación, en cambio, se asemejan a los miembros de la primera generación nacida en un país nuevo. Debemos aprender junto con los jóvenes la forma de dar los próximos pasos; pero para proceder así, debemos reubicar el futuro.

¿Será que todavía estamos atados a concepciones tradicionales del aprendizaje, aquellas con las que aprendimos? Desde luego, es necesario tener claro que las TIC tienen que servir para innovar procesos educativos, permitir al estudiante ser sujeto activo en la construcción de su aprendizaje, brindar espacios para que investigue, construya, reflexione, plantee.

Por lo anterior, el modelo de profesor o profesora que posee el conocimiento y lo transmite a sus estudiantes está obsoleto. En la actualidad, es necesario trabajar con los y las jóvenes en el desarrollo de competencias requeridas para sobrevivir en la Sociedad de la Información y el Conocimiento: capacidad de síntesis, lectura crítica, selección de información, de manera que pueda transformar los datos, la información en conocimiento. 
Por lo demás, en muchos casos, y al usar tecnología, nos encontramos con que nuestra población estudiantil sabe más que nosotros y nosotras. Esto no tiene que ser una limitante para introducir estas herramientas como medios para innovar procesos educativos; parafraseando a Mead (1971, citada por Martín-Barbero (2002), es necesario, aprender junto con los y las jóvenes a dar los primeros pasos en la construcción de ambientes educativos realmente innovadores, donde las posibilidades de las TIC sí impacten el medio educativo.

\subsection{El uso de las bitácoras electrónicas "blogs" como estrategia para el desarrollo de la competencia comunicativa}

A continuación, quiero compartir con ustedes mis aprendizajes con el uso de las bitácoras electrónicas "blogs" como estrategia para el desarrollo de la competencia comunicativa.

Esta reseña recoge las principales enseñanzas de una experiencia puesta en práctica en el primer semestre del 2008 con veintitrés estudiantes (cinco varones y dieciocho mujeres) del curso Expresión Escrita I del Bachillerato en Educación Primaria de la Escuela de Formación Docente de la Facultad de Educación de la Universidad de Costa Rica.

Así pues, el enfoque comunicativo en la enseñanza de la lengua prioriza el aprendizaje del uso verbal en contextos significativos para el aprendiz (Lomas [comp.], 1996; Lomas, Osoro y Tusón, 1993); asimismo, Vigotsky plantea que: "el lenguaje y el pensamiento tienen origen social y se transmiten y se desarrollan a partir de la interacción contextualizada entre hablantes" (Tudge, 1993); por su parte Cassanny (1999), se refiere a las nuevas prácticas de los y las jóvenes, tales como viajar por la internet en busca de datos, utilizar programas de edición de textos, correos electrónicos, conversaciones sincrónicas, "Chat", MSN, y nos plantea la posibilidad de que los aprendices actuales se puedan sentir desmotivados y desmotivadas por seguir aprendiendo a escribir en soportes que ya no utilizan.

Por estas razones pensé en los "blogs" como una posibilidad para generar un contexto significativo para estos y estas estudiantes, quienes recién han concluido sus estudios secundarios y se encuentran en el primer semestre de su vida universitaria. De este modo, un "blog" es un sitio web fácil de usar, en donde se puede, entre muchas otras actividades, expresar rápidamente la opinión e interactuar con otros usuarios. 
Ahora bien, Expresión Escrita I forma parte del plan de estudios del Bachillerato en Educación Primaria; este curso debe aprobarse en el primer semestre de la carrera. En el programa oficial del curso, en la descripción del mismo, se plantea lo siguiente

Su finalidad es sentar las bases didácticas de la expresión escrita. Por tanto, se propone dotar al alumno de los conocimientos teóricos y prácticos necesarios para la adquisición de las habilidades y destrezas que demanda el proceso de construcción y desciframiento de eficaces mensajes escritos que transmitan a cabalidad el pensamiento y faciliten la interacción social. El desarrollo adecuado de esta competencia lingüística estimula en los futuros docentes el trabajo creativo de la composición y la redacción, y les permite generar experiencias propias en el campo, a fin de preparase para guiar mejor el proceso de aprendizaje en los primeros dos ciclos de la educación general básica.

Por lo anterior, en el primer semestre del 2008, como parte de las actividades de aprendizaje planteadas para este curso, se propuso el trabajo con blogs; para ello, en la primera sesión del curso, dediqué una parte de la lección para que ingresaran a http://www.blogger.com/ y les di una explicación básica sobre su funcionamiento; el resto fue explorar en conjunto. Según lo manifestado por ellos y ellas, hasta ese momento exploraban el recurso; sin embargo, no tuvieron mayores dificultades en su uso; por el contrario, dieron rienda suelta a su creatividad, tuvieron la oportunidad de explorar las posibilidades que brinda el texto electrónico de combinar información visual, auditiva y lingüística.

A partir de lo anterior, concebí el curso como un espacio para la producción de texto, pues para lograr realizar esta tarea con éxito se necesita, no solo contar con las destrezas necesarias para el desarrollo de la competencia lingüística y comunicativa, sino también tener conocimiento del mundo en donde vivimos y de las problemáticas en torno a él; por lo anterior, las sesiones de trabajo se organizaron combinando la revisión de los contenidos teóricos planteados en el programa con el desarrollado del siguiente tema: "Centroamérica hoy: globalización, pobreza y desigualdad", como punto de partida para generar discusión y construcción de conocimiento, a través de estrategias para la producción de texto escrito en lengua materna.

En concordancia con lo planteado, en las sesiones de trabajo se llevaron a cabo: exposiciones magistrales, discusiones grupales, exposiciones de los estudiantes, conversaciones con expertos en la temática planteada. Con el objetivo de aprovechar el 
potencial que brindan la internet, y sobre todo la Web 2.0, semanalmente se contaba con un espacio de 60 minutos en el laboratorio de informática en donde se exploraban las posibilidades que brindan los "blogs" para la producción escrita en la internet.

Se formaron ocho subgrupos y cada subgrupo debía elegir un tema de investigación en torno a la temática del curso. Antes de que cada subgrupo eligiera el subtema de investigación se propusieron lecturas y reflexiones en torno a la temática; los subtemas fueron surgiendo de esas reflexiones y fueron planteados por el estudiantado; estos fueron los siguientes:

- Sistemas Políticos Centroamericanos.

- Sistemas Educativos Centroamericanos.

- Violencia en Centroamérica.

- Panamá, un ejemplo de desarrollo.

- Analfabetismo en Centroamérica.

- Sistemas de Salud en Centroamérica.

- Pobreza en Centroamérica.

- Los situación de los recursos naturales en Centroamérica.

Cada subgrupo debía elaborar un blog como un medio para difundir los resultados de su investigación exploratoria sobre el tema seleccionado; esto sirvió para fomentar la interacción entre ellos y ellas. Las direcciones electrónicas debían intercambiarse entre todos los subgrupos, de esta manera, se fomentaba el acceso a los diferentes blogs, todos y todas podían revisar los de los otros subgrupos.

Constantemente se intercambiaban conocimientos sobre la elaboración de estos, ya que cuando revisaban y descubrían alguna nueva posibilidad, ya explorada por un subgrupo, surgían preguntas y a partir de las respuestas, trataban de aplicarlo en su "blog"; se invitaron a personas externas a leer y a escribir comentarios: amigos y amigas de los y las integrantes del subgrupo, amigos, amigas y mis colegas se interesaron en la experiencia.

Esta práctica generó la posibilidad de perfeccionar la expresión escrita en contextos significativos, y los y las estudiantes tuvieron oportunidad de interactuar alrededor de una temática de gran actualidad. Como resultado, se obtuvieron ocho bitácoras electrónicas, que se encuentran disponibles en las siguientes direcciones ${ }^{4}$ :

- http://www.grupoexpresion1.blogspot.com/

- http://www.fabileo7.blogspot.com/

4 Se invita al lector o lectora visitar los "blogs". 
- http://www.grupo3expre.blogspot.com/

- http://www.expresionescritai.blogspot.com/

- http://www.mentesbrillantespca.blogspot.com/

- http://expresioncentroamericahoy.blogspot.com/

- $\quad$ http://www.lomejordeeducacion.blogspot.com/

- http://www.kamabren.blogspot.com

\subsection{Evaluación de la experiencia por parte del estudiantado}

En las valoraciones hechas por los y las estudiantes del curso sobre esta experiencia ${ }^{5}$ se calificó como: interesante, novedosa, agradable, entretenida, creativa, interactiva e instructiva; además, se valoró la posibilidad que esta herramienta brinda para que las clases fueran más amenas; también, se consideró una forma diferente, constructiva y original de aprender y como una forma de romper con esquemas tradicionalistas; asimismo, se valoró la posibilidad que brinda de introducir al estudiantado en el mundo de la tecnología.

A su vez, se destacó la posibilidad que brinda para almacenar información, transmitir la manera de pensar; afirmaron que fomenta la participación y la reflexión, que ayuda a difundir información de forma interactiva, ya que está a disposición de cualquier persona que quiera accesarla.

Además, se afirma que ayudó a fomentar el trabajo colaborativo, ya que lo que un compañero o compañera sabía, lo compartía con los demás; también se consideró que ayudó a descubrir nuevas formas de utilizar las computadoras y es una forma de desarrollar las habilidades, utilizando recursos tecnológicos y, por último, se valoró como una herramienta de investigación que ayudó a aprender mucho.

\section{CONCLUSIONES}

La experiencia llevada a cabo, durante el primer semestre del 2008 con los y las estudiantes del Curso Expresión Escrita 1 del Bachillerato en Educación Primara de la Escuela de Formación Docente de la Facultad de Educación de la Universidad Costa Rica, me permite afirmar que, efectivamente, las bitácoras electrónicas constituyen una herramienta muy valiosa para democratizar los servicios de la internet, así como para animar

5 Se invita al lector o lectora a consultar las evaluaciones en la siguiente dirección electrónica: http://expresionescrita1.blogspot.com/2008/06/opiniones-sobre-el-uso-de-los-blog.html 
las prácticas educativas y acercar la comunidad estudiantil al uso de herramientas gratuitas disponibles en la web, y de gran utilidad para fomentar el desarrollo de la competencia lingüística y comunicativa en contextos significativos para ellos y ellas.

En ese sentido, la herramienta, aunque no era conocida, resultó fácil de aprender, dinámica, entretenida, generadora de un proceso de aprendizaje constructivo, innovador y ayudó a fomentar el aprendizaje colaborativo.

En el fondo, la Web 2.0 constituye, sin duda, un reto para la educación formal; las posibilidades que brinda aún no han sido exploradas suficientemente en la educación. Al respecto, y para finalizar, quiero rescatar literalmente, una de las valoraciones hechas sobre esta experiencia y que textualmente expresó: "no solo darles un uso recreativo y nada más".

He ahí el reto, que estas herramientas no constituyan solo una práctica de socialización o recreación, sino que con la misma naturalidad y espontaneidad que son utilizadas por los y las jóvenes para comunicarse, socializar y entretenerse, sean utilizadas como parte del sistema educativo formal.

\section{Referencias}

Cassany, Daniel. (1999). Construir la escritura. Barcelona: Paidós Ibérica S.A.

Castells, Manuel (2006). La Sociedad Red: Una Visión Global. España: Alianza Editorial

Fonseca, Clotilde. (2000) "Maestros Innovadores: El Papel de la Informática Educativa en el desarrollo profesional de los educadores costarricenses". V Foro Educativo "La nueva educación-Informática educativa en la educación del tercer milenio". Bogotá, Colombia, 12-13 de octubre. Recuperado el 10 de agosto 2008 de http://www.fod.ac.cr/fuentes/MaestrosInnovadoresPapeldelalnform\%E1 ticaEducativa.p $\underline{\mathrm{df}}$

Hernández, Pedro. (2007). Tendencias de Web 2.0 aplicadas a la educación en línea. No Solo Usabilidad journal, (6). Recuperado el 13 de febrero de 2010 de http://www.nosolousabilidad.com/articulos/web20.htm

Instituto de Estudios Sociales en Población (2009). Población joven, TICs e Interes Políticos. Dos estudios de percepciones. Recuperado el 2 de octubre de 2009. http://www.una.ac.cr/idespo/pdf/PRESENTACION IDESPO JOVENES.pdf

Lomas, Carlos y Osoro, Andrés. (1993). El enfoque comunicativo de la enseñanza de la lengua. Barcelona, España: Paidós 
Lomas Carlos. (1996). La educación lingüística y literaria en la enseñanza secundaria. Barcelona, España: ICE / Horsoni

Martín-Barbero, Jesús. (2002). Jóvenes: comunicación e identidad. Pensar Iberoamérica. No.0 .Febrero. http://www.oei.es/pensariberoamerica/ric00a03.htm

Proyecto Estado de la Nación (2008). Resumen Estado de La Nación en Desarrollo Humano Sostenible: XIV informe. San José, Costa Rica. Recuperado el 1 de marzo de 2010, de http://www.estadonacion.or.cr/Info2008/Paginas/equidad.html\#Educacion

Proyecto Estado de la Nación. (s.f.). Deserción juvenil en las aulas. Actividad cuatro. $\begin{array}{llllll}\text { Recuperado el } & 10 & \text { de } & \text { Agosto } & \text { del }\end{array}$ www.estadonacion.or.cr/Publicaciones/Des sost sec/121-132.pdf

Serrano, Jorge. (2006). Pasado, presente y futuro de la Web 2.0 en servicios de información digital. Bid. Textos Universitaris de Biblioteconoma i Documentació, (17). Recuperado el 13 de febrero de 2010 de http://www.ub.edu/bid/17serra2.htm

Tudge, Jonathan. (1993). La zona de desarrollo próximo y la colaboración entre pares: connotaciones para la práctica del aula. En Luis C. Moll (Comp.), Vygotsky y la educación. Buenos Aires, Argentina: Aique Grupo Editor S. A.

Universidad de Costa Rica. Escuela Formación Docente. (2008). Programa del Curso- FD1015 La Expresión Escrita en la Educación Primaria I. I-2008. San Pedro: UCR

Universidad de Costa Rica. Programa Sociedad de la Información y el Conocimiento. (2008a). Los jóvenes costarricenses en la Sociedad de la Información. Avance de Resultados. Recuperado el 01 de Agosto del 2008, de http://www.prosic.ucr.ac.cr

Universidad de Costa Rica. Programa Sociedad de la Información y el Conocimiento. (2008b). Los jóvenes costarricenses en la Sociedad de la Información. Informe de Resultados 2008. Recuperado el 10 de diciembre de 2009, de http://www.prosic.ucr.ac.cr 